
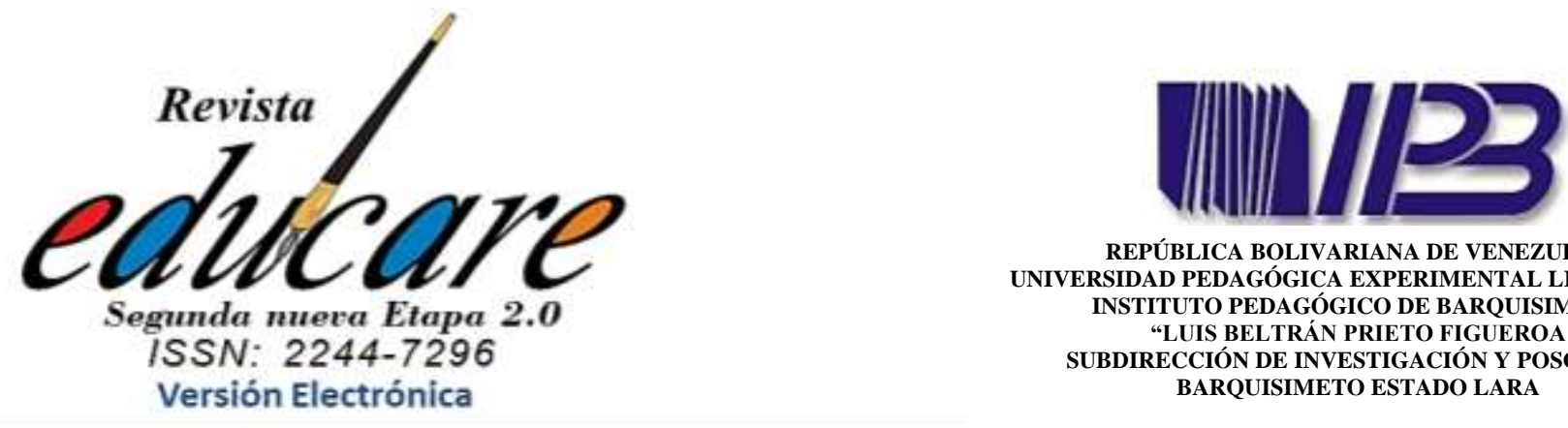

REPÚBLICA BOLIVARIANA DE VENEZUELA UNIVERSIDAD PEDAGÓGICA EXPERIMENTAL LIBERTADOR INSTITUTO PEDAGÓGICO DE BARQUISIMETO "LUIS BELTRÁN PRIETO FIGUEROA SUBDIRECCIÓN DE INVESTIGACIÓN Y POSGRADO BARQUISIMETO ESTADO LARA

Volumen 24 № 1 Enero-Abril 2020

(98-111)

Eva Katherine Macías García ORCID: https://orcid.org/0000-0003-2739-8648

Gladys María Saltos Briones ORCID: https://orcid.org/0000-0002-6668-1668

UNIVERSIDAD TÉCNICA DE MANABí

*Economista, estudiante de la Maestría en Administración de Empresas en el Instituto de Postgrado de la Universidad Técnica de Manabí, Ecuador. Actualmente trabaja como Secretaria de la Carrera de Ingeniería Química de la Universidad Técnica de Manabí, evakmg@ hotmail.com

** Doctora en Ciencias Económicas. Actualmente se desempeña como Docente de la Universidad Técnica de Manabí en la Facultad de Ciencias Administrativas y Económicas, Ecuador, gsaltos@utm.edu.ec

\section{INCIDENCIA DEL CLIMA ORGANIZACIONAL EN EL DESEMPEÑO LABORAL DE LA CARRERA DE INGENIERÍA QUÍMICA DE LA UNIVERSIDAD TÉCNICA DE MANABÍ}

Recibido:

06-11-2019

Aceptado:

28-01-2020
INCIDENCE OF THE ORGANIZATIONAL CLIMATE IN THE LABOR PERFORMANCE OF CHEMICAL ENGINEERING CAREER OF THE UNIVERSIDAD TÉCNICA DE MANABÍ 

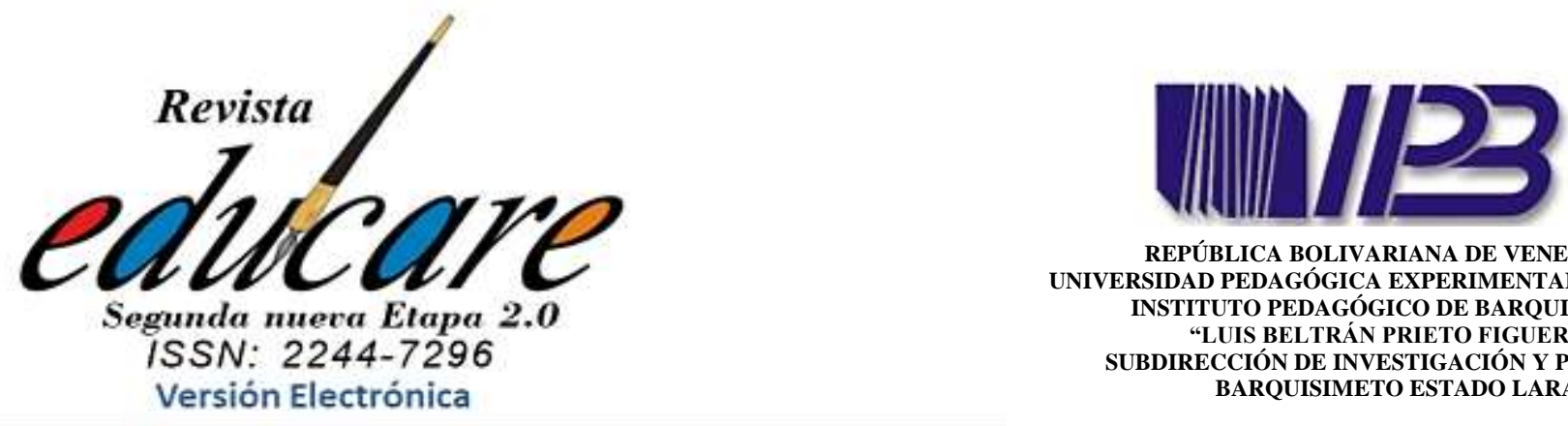

REPÚBLICA BOLIVARIANA DE VENEZUELA UNIVERSIDAD PEDAGÓGICA EXPERIMENTAL LIBERTADOR INSTITUTO PEDAGÓGICO DE BARQUISIMETO "LUIS BELTRÁN PRIETO FIGUEROA SUBDIRECCIÓN DE INVESTIGACIÓN Y POSGRADO BARQUISIMETO ESTADO LARA

\section{Resumen}

El funcionamiento eficiente de las organizaciones depende del clima organizacional, cuyo análisis permite revelar factores que afectan su productividad, mientras que el desempeño laboral incide directamente sobre los resultados de la organización. Esta investigación tiene por objeto el análisis del clima organizacional y su influencia en el desempeño laboral para elaborar una serie de recomendaciones que sirvan de apoyo en la construcción de un plan de mejoras; se hará mediante una investigación descriptiva, realizándose un diagnóstico situacional mediante la observación directa y la aplicación de cuestionarios a los docentes de la carrera de Ingeniería Química de la Universidad Técnica de Manabí. Los resultados permitieron elaborar recomendaciones para el plan de mejora que, de ser tomado en cuenta, redundará positivamente en la calidad del servicio que esta dependencia brinda a la comunidad universitaria, mejorando el clima organizacional y por ende el desempeño laboral de sus integrantes.

Descriptores. carrera de Ingeniería Química de la UTM; clima organizacional; desempeño laboral.

\section{Abstract}

The efficient functioning of organizations depends on the organizational climate, whose analysis reveals factors that affect their productivity, while work performance directly affects the results of the organization. This research aims at analyzing the organizational climate and its influence on labor performance to develop a series of recommendations that will support the construction of an improvement plan; It will be done through descriptive research, with a situational diagnosis being carried out by direct observation and the application of questionnaires to the teachers of the Chemical Engineering degree of the Technical University of Manabí. The results made it possible to draw up recommendations for the improvement plan, which, if taken into account, will positively result in the quality of the service that this unit provides to the university community, improving the organizational climate and thus the work performance of its members.

Keywords: UTM Chemical Engineering Career; organizational climate; job performance. 


\section{INTRODUCCIÓN}

El ambiente que se genera en las organizaciones es indicio de cómo interactúan las características personales y las de la organización; este ambiente o clima comprende a la organización como tal, con su estructura y funcionamiento, el lugar donde las personas realizan el trabajo y las actividades que realizan, constituyendo así un sistema muy dinámico e interdependiente que influye en la productividad de los trabajadores (Pérez et al., 2009).

Debido a esta influencia, es labor de las organizaciones buscar el bienestar de sus trabajadores, para que de esta manera el servicio aportado por ellos sea de calidad e incida positivamente en la consecución de los objetivos que apunten hacia el éxito organizativo. Este bienestar se genera a través de un buen clima organizacional, sin embargo, en gestión del recurso humano, su estudio constituye un tema complejo al convertirse éste en un tema de alta relevancia por ser la forma más efectiva de diagnosticar la satisfacción del personal y su compromiso con los objetivos trazados por la empresa (Vera y Suárez, 2018).

A nivel mundial, puede observarse que en las empresas que alcanzan logros de gran importancia, el clima organizacional es un aspecto muy tomado en cuenta y de especial relevancia que permite alcanzar los objetivos formulados. Sin embargo, en América Latina, las organizaciones implementan políticas rígidas e incluso obsoletas para el manejo de estos ambientes; en Ecuador, la mayoría de las empresas gubernamentales utilizan estos métodos en los que el personal no es tomado en cuenta para la mejora del servicio sino como ente productor (Salinas, 2015).

En universidades ecuatorianas, ya se han realizado estos análisis, resultando que uno de los factores de mayor riesgo obtenidos del análisis del clima organizacional es el factor remuneración, con un porcentaje de satisfacción de apenas el 38.9, seguido de la capacitación y desarrollo (44.8\%), la compensación y el reconocimiento (45.7\%), la comunicación (47\%), la normatividad y procesos (48.5), el trabajo en equipo (57.5\%) y la motivación y expectativas (58.5\%), entre otros (Quiñónez, 2013).

El hacer un diagnóstico de estos factores de riesgo en las universidades, sirve no solo a los integrantes de un departamento sino, en el caso de estudio, a toda la comunidad universitaria, 
pudiéndose replicar este estudio en otros o aplicar las recomendaciones emanadas de los resultados de la investigación.

La finalidad de esta investigación, es la de hacer un diagnóstico del clima organizacional y su incidencia en el desempeño laboral en la carrera de Ingeniería Química de la Facultad de Ciencias Matemáticas, Físicas y Químicas de la Universidad Técnica de Manabí.

\section{REFERENTES TEÓRICOS}

A continuación, se darán algunas concepciones teóricas consideradas necesarias para el desarrollo de la investigación.

\section{Clima organizacional}

Según Vega, el clima organizacional,

“... describe una organización, la distingue de otras e influye en el comportamiento de las personas que la conforman; además, agrupa aspectos organizacionales tales como las prácticas, las políticas, el liderazgo, los conflictos, los sistemas de recompensas y de castigos, el control y la supervisión, así como las particularidades del medio físico de la organización”. (Vega et al., 2006:337)

El hombre vive en un ambiente complejo y además dinámico, y de allí nace la idea de clima organizacional al estar las organizaciones compuestas por colectividades, grupos, personas que generan comportamientos diversos y que afectan ese ambiente (García, 2009).

Los componentes que inciden en el clima organizacional son:

. Ambiente físico: comprende el espacio físico, las instalaciones, los equipos instalados, el color de las paredes, la temperatura, el nivel de contaminación, entre otros.

. Características estructurales: como el tamaño de la organización, su estructura formal, el estilo de dirección, etcétera.

- Ambiente social: que abarca aspectos como el compañerismo, los conflictos entre personas o entre departamentos, la comunicación y otros.

- Características personales: como las aptitudes y las actitudes, las motivaciones, las expectativas, etcétera.

- Comportamiento organizacional: compuesto por aspectos como la productividad, el ausentismo, la rotación, la satisfacción laboral, el nivel de tensión, entre otros (Salazar et al., 2009:69). 
En la educación superior, el clima organizacional puede ser conocido también como clima universitario, clima institucional, clima educativo, clima laboral o clima organizacional y está determinado por sus objetivos de estudio y por las orientaciones de los investigadores. Cuando se trata de ambiente educativo, el clima está constituido por el ambiente interno, por el contexto, el comportamiento y el enfoque estructural que tenga la organización. La gestión en los diferentes niveles dentro de una universidad debe constituir una responsabilidad y un compromiso también para los directivos, quienes deben actuar con determinación con el fin de establecer la calidad como valor fundamental debiendo ser incorporado a las normas administrativas (González y Pérez, 2007). El clima organizacional resulta una herramienta administrativa y un enfoque importante para la toma de decisiones, permitiendo proyectar el aumento de la productividad e inducir cambios (Segredo, 2013).

\section{Desempeño laboral}

El desempeño laboral es el comportamiento que tiene un empleado en la consecución de los objetivos fijados, lo que constituye una estrategia individual para lograrlos; inciden características como habilidades, capacidades, cualidades y necesidades que interactúan con la naturaleza del trabajo y también de la organización, produciendo comportamientos que afectan los resultados (Quintero et al., 2008).

También, se puede decir que el desempeño laboral "... describe el grado en que los gerentes o coordinadores de una organización logran sus funciones, tomando en cuenta los requerimientos del puesto ocupado, con base a los resultados alcanzados". (Araujo y Guerra, 2007).

Hay elementos que inciden en el desempeño laboral y que se relacionan y generan un alto o bajo desempeño laboral. Entre estos elementos se tienen la satisfacción en relación con las tareas asignadas, las retribuciones monetarias y no monetarias, las competencias para realizar las tareas asignadas, la capacitación y desarrollo constante de los empleados, las expectativas del empleado, los factores motivacionales y conductuales del individuo, la cultura organizacional y el clima organizacional (Palaci, 2005).

El desempeño es pues, un elemento fundamental para que una organización funcione, debiéndosele prestar atención especial dentro de los procesos administrativos de sus recursos humanos. Constituye el comportamiento del evaluado en su proceso por lograr los objetivos asignados, formando parte de su estrategia individual para lograrlos (Pedraza et al., 2010). 


\section{MATERIALES Y MÉTODOS}

El tipo de investigación es descriptiva ya que consiste en la compilación de información que describe un acontecimiento para luego ser organizada, tabulada y representada; se apoya en gráficos y tablas que facilitan el entendimiento de la distribución de los datos que han sido recopilados (Abreu, 2012). Se buscará información sobre el clima organizacional de la carrera de Ingeniería Química para determinar su incidencia en el desempeño laboral de los involucrados.

El diseño de investigación es cualitativo cuyo enfoque es inductivo (de lo particular a lo general), iniciándose con una suposición (Sousa et al., 2007). Se establecerá una relación entre los datos recopilados en los instrumentos de recolección de datos y la observación, sobre la base de los cálculos producto de su procesamiento.

El estudio se realizará sobre toda la población por lo que no hay muestra; la población representa al conjunto de individuos de los que se desea conocer algo, también denominado universo (López, 2004). Está conformada por 20 unidades estadísticas o componentes de la población (Torres et al., 2014), entre los que se encuentran el Vicedecano de la carrera de Ingeniería Química, el Coordinador y 18 docentes.

Los instrumentos de recolección de datos son mecanismo para recabar y registrar información pertinente (Corral, 2009); los usados fueron el cuestionario, expresado en una serie de preguntas diseñadas con antelación por los investigadores y redactadas a partir de las preguntas de investigación (Martínez, 2013), además de la observación directa. Las preguntas están basadas en tres dimensiones: general, clima organizacional y desempeño laboral.

El cuestionario contiene preguntas cerradas (de selección y dicotómicas), preguntas con escalas de satisfacción y abiertas. Para su análisis, en algunos casos se usó la semaforización (0 - 20\% - Critico, 21 - 40\% - Riesgo, 41 - 60\% - Preventivo, 61 - 80\% - Adecuado, 81 - 100\% Óptimo), en otros la mayor incidencia y categorización. Se utilizó el programa SPSS para el procesamiento de los datos.

El procedimiento de la investigación ha sido:

- Revisión de los referentes teóricos que sirvan de sustento para darle cientificidad a la investigación;

. Definición del objetivo central; 
. Definición de las preguntas de investigación que orientarán la consecución del objetivo;

- Realización de un diagnóstico situacional del caso en estudio, mediante la creación y revisión de instrumentos de recolección de datos, su aplicación y procesamiento;

. Análisis de los datos;

. Elaboración de una serie de recomendaciones como insumos para un plan de mejoras con incidencia positiva en el desempeño laboral de los integrantes de la carrera de Ingeniería Química.

\section{RESULTADOS Y DISCUSIÓN}

Una vez procesados los datos en cuanto a la dimensión de información general, se ha detectado que la mayoría de los docentes es de categoría auxiliar (30\%), seguido de principal con un 25\%; todos tienen una dedicación a tiempo completo y el 35\% han trabajado en la institución entre 1 y 5 años, al igual que los que tienen más de 10 años de antigüedad. Solo el $15 \%$ es contratado.

Llama la atención que el $75 \%$ son de sexo masculino, al igual que un estudio hecho en la carrera de Ingeniería Química de la Universidad Central de Ecuador, quienes en una investigación realizada desde su primera promoción en el año 1954 hasta el 2008, detectaron que el 75\% lo representan hombres (Universidad Central del Ecuador, 2009).

Los resultados de la dimensión de clima organizacional pueden apreciarse en las tablas 1 y 2.

Tabla 1: Preguntas de la dimensión clima organizacional (mayor incidencia y categorización)

\begin{tabular}{|l|l|}
\hline \multicolumn{1}{|c|}{ Motivación } & Porcentaje \\
\hline Crecimiento profesional & $40 \%$ \\
\hline Mejora del salario & $25 \%$ \\
\hline Ascenso / Promoción & $20 \%$ \\
\hline Buen ambiente, buen trato, buena comunicación & $5 \%$ \\
\hline
\end{tabular}

Fuente: elaboración propia. 
Tabla 2: Preguntas de la dimensión clima organizacional (semaforización y categorización)

\begin{tabular}{|c|c|c|}
\hline Factor & $\begin{array}{l}\text { Valoració } \\
\mathrm{n}\end{array}$ & Lectura \\
\hline Estilo de dirección (Muy aceptable) - Decanato & $80 \%$ & Adecuado \\
\hline Estilo de dirección (Muy aceptable) - Vicedecanato & $90 \%$ & Óptimo \\
\hline Estilo de dirección (Muy aceptable) - Coordinación & $90 \%$ & Óptimo \\
\hline $\begin{array}{l}\text { Estilo de dirección (Muy aceptable) - Encargados } \\
\text { de investigación, vinculación y otros }\end{array}$ & $75 \%$ & Adecuado \\
\hline Clima laboral (Muy competitivo) & $45 \%$ & Preventivo \\
\hline Compañerismo (Muy agradable) & $25 \%$ & Riesgo \\
\hline Relación con (Muy cordial) - Decanato & $90 \%$ & Óptimo \\
\hline Relación con (Muy cordial) - Vicedecanato & $90 \%$ & Óptimo \\
\hline Relación con (Muy cordial) - Coordinador & $95 \%$ & Óptimo \\
\hline $\begin{array}{l}\text { Relación con (Muy cordial) - Encargados de } \\
\text { investigación, vinculación y otros }\end{array}$ & $70 \%$ & Adecuado \\
\hline $\begin{array}{l}\text { Evaluación contribuye a mejorar debilidades } \\
\text { (Totalmente de acuerdo) }\end{array}$ & $55 \%$ & Preventivo \\
\hline Motivación (Totalmente de acuerdo) - Decanato & $40 \%$ & Riesgo \\
\hline $\begin{array}{l}\text { Motivación (Totalmente de acuerdo) - } \\
\text { Vicedecanato }\end{array}$ & $45 \%$ & Preventivo \\
\hline $\begin{array}{l}\text { Motivación (Totalmente de acuerdo) - } \\
\text { Coordinación }\end{array}$ & $45 \%$ & Preventivo \\
\hline $\begin{array}{l}\text { Motivación (Totalmente de acuerdo) - Encargados } \\
\text { de investigación, vinculación y otros }\end{array}$ & $30 \%$ & Riesgo \\
\hline Está de acuerdo como es evaluado - docencia & $95 \%$ & Óptimo \\
\hline $\begin{array}{l}\text { Está de acuerdo como es evaluado - actividades } \\
\text { administrativas, de gestión, de investigación y otras }\end{array}$ & $95 \%$ & Óptimo \\
\hline Criterios en la evaluación de desempeño & $95 \%$ & Óptimo \\
\hline $\begin{array}{l}\text { Uso de herramientas apropiadas para evaluar el } \\
\text { desempeño }\end{array}$ & $95 \%$ & Óptimo \\
\hline
\end{tabular}

Fuente: elaboración propia.

Llama la atención, que los docentes de la carrera de Ingeniería Química han seleccionado como mayor motivación su crecimiento profesional; y, es que, a pesar de que resulta una motivación esencial para su desempeño laboral, también forma parte de la autorrealización. La autorrealización es fuente, además de motivación, de compromiso. Proporcionar este tipo de satisfacción constituye un camino seguro al alto rendimiento organizacional. 
En cuanto a las valoraciones de satisfacción, hay tres parámetros que están en estado de riesgo, lo que implica que el clima organizacional está en mal estado en estos sentidos y que se deben de tomar acciones a corto plazo. Estos son el compañerismo, la motivación por parte del Decanato, y la motivación por parte de los encargados de las áreas de investigación, vinculación, evaluación y otros.

Se encontraron parámetros en estado preventivo, indicando que el clima organizacional está comenzando a deteriorarse y que hay que tomar acciones a mediano plazo. Estos son, el clima laboral que debe ser más competitivo, el hecho de que las evaluaciones deben perfilarse más hacia la mejora de debilidades y la motivación por parte del Vicedecanato y la Coordinación.

Los resultados de la dimensión desempeño laboral pueden apreciarse en las tablas 3 y 4.

Tabla 3: Preguntas de la dimensión desempeño laboral (mayor incidencia y categorización)

\begin{tabular}{|l|c|c|c|c|}
\cline { 2 - 5 } \multicolumn{1}{c|}{} & Temperatura & Ergonomía & Ruido & Incidencia \\
\hline $\begin{array}{l}\text { Mejoras al espacio - } \\
\text { docencia }\end{array}$ & $70 \%$ & $25 \%$ & $5 \%$ & $\begin{array}{c}\text { Oscurecer } \\
\text { las ventanas (1) }\end{array}$ \\
\hline $\begin{array}{l}\text { Mejoras al espacio - } \\
\text { actividades administrativas, } \\
\text { de gestión, de investigación y } \\
\text { otras }\end{array}$ & $70 \%$ & $15 \%$ & $5 \%$ & $\begin{array}{l}\text { Impresora } \\
\text { para libros (1) }\end{array}$ \\
\hline
\end{tabular}

Fuente: elaboración propia.

Tabla 4: Preguntas de la dimensión desempeño laboral (semaforización y categorización)

\begin{tabular}{|l|c|c|}
\hline \multicolumn{1}{|c|}{ Factor } & Valoración & Lectura \\
\hline Espacio físico (Muy agradable) - actividades de docencia & $45 \%$ & Preventivo \\
\hline $\begin{array}{l}\text { Espacio físico (Muy agradable) - actividades } \\
\text { administrativas, de gestión, de investigación y otras }\end{array}$ & $30 \%$ & Riesgo \\
\hline $\begin{array}{l}\text { Capacitación permite innovar (Totalmente de acuerdo) - } \\
\text { actividades de docencia }\end{array}$ & $65 \%$ & Adecuado \\
\hline $\begin{array}{l}\text { Capacitación permite innovar (Totalmente de acuerdo) - } \\
\text { actividades administrativas, de gestión, de investigación y } \\
\text { otras }\end{array}$ & $65 \%$ & Adecuado \\
\hline Cuenta con insumos - actividades de docencia & $60 \%$ & Preventivo \\
\hline Cuenta con insumos - actividades administrativas, de & $65 \%$ & Adecuado \\
\hline
\end{tabular}


Eva Katherine Macías García y Gladys María Saltos Briones

\begin{tabular}{|l|c|l|}
\hline gestión, de investigación y otras & & \\
\hline Existen condiciones - actividades de docencia & $90 \%$ & Óptimo \\
\hline $\begin{array}{l}\text { Existen condiciones - actividades administrativas, de } \\
\text { gestión, de investigación y otras }\end{array}$ & $70 \%$ & Adecuado \\
\hline $\begin{array}{l}\text { Distribución de carga académica repercute - actividades } \\
\text { de docencia }\end{array}$ & $45 \%$ & Preventivo \\
\hline $\begin{array}{l}\text { Distribución de carga académica repercute - actividades } \\
\text { administrativas, de gestión, de investigación y otras }\end{array}$ & $55 \%$ & Preventivo \\
\hline $\begin{array}{l}\text { Ambiente cubre expectativas } \\
\text { Conoce lo que esperan de - actividades de docencia }\end{array}$ & $100 \%$ & Óptimo \\
\hline $\begin{array}{l}\text { Conoce lo que esperan de - actividades administrativas, } \\
\text { de gestión, de investigación y otras }\end{array}$ & $100 \%$ & Óptimo \\
\hline
\end{tabular}

Fuente: elaboración propia.

Un factor que incide de forma drástica en el desempeño laboral lo representa la mejora al espacio tanto en el área de la docencia como la destinada a las actividades administrativas, de gestión, investigación y otras. Estas mejoras están dirigidas a la temperatura, tomando en cuenta que la carrera funciona en la ciudad de Portoviejo (Ecuador), y que en el año 2019 se registró en el boletín de diciembre del Instituto Nacional de Meteorología e Hidrología temperaturas máximas de $31.5^{\circ} \mathrm{C}$ con sensaciones térmicas superiores (Instituto Nacional de Meteorología e Hidrología, 2019); esto sin considerar que el verano está comprendido entre los meses de junio a diciembre, tiempo en el que las temperaturas son más bajas.

Los ambientes calurosos pueden generar estrés térmico provocando una disminución en el rendimiento y posibles daños a la salud (Camacho, 2013). Las afectaciones podrían ser golpes de calor, agotamiento por el calor, deshidratación, entre otros. Los ambientes con temperaturas inadecuadas restan confort a los empleados ya que el cuerpo humano dedica gran parte de su energía a regular la temperatura corporal, disminuyendo la energía que debe dedicar a la concentración, creatividad e inspiración.

En cuanto a las valoraciones de satisfacción, hay un parámetro que está en estado de riesgo, lo que implica que el desempeño laboral es bajo debido en parte a este rubro y que se deben de tomar acciones a corto plazo. Este indicador es el espacio físico destinado a las actividades administrativas, de gestión, de investigación y otras. 
Se encontraron parámetros en estado preventivo, indicando que el desempeño laboral es de nivel promedio en ese sentido y que hay que tomar acciones a mediano plazo. Estos son los espacios físicos destinados a la docencia, los insumos disponibles para las actividades de docencia, y la repercusión negativa de la carga académica en las actividades de docencia, administración, gestión, investigación y otras.

\section{CONCLUSIONES}

Al finalizar esta investigación se ha llegado a las siguientes conclusiones:

. El clima organizacional es el ambiente laboral que una institución tiene al interno y que está relacionado con las emociones de sus integrantes y estrechamente relacionado con la motivación.

. El desempeño laboral es el parámetro que indica el rendimiento laboral de los integrantes de una organización, además de su actuación mientras desarrolla las funciones relacionadas a su cargo.

. La motivación que se ejerce mediante la planificación del crecimiento profesional de los docentes, además de ser parte de su autorrealización, es fuente de motivación y compromiso, incidiendo en un alto rendimiento organizacional.

. En el clima organizacional del caso de estudio, se encontraron tres factores de riesgo que son el compañerismo, la motivación por parte del Decanato, y la motivación por parte de los encargados de las áreas de investigación, vinculación, evaluación y otros. Hay tres factores en estado de prevención, siendo estos la necesidad de mayor competitividad entre los compañeros docentes, los resultados de las evaluaciones que deben enfocarse en superar las debilidades, y la necesidad de mejorar la motivación por parte del Vicedecanato y Coordinación.

. En cuanto al desempeño laboral, el espacio físico es de vital importancia, encontrándose que deben tomarse acciones inmediatas en cuanto a la regulación de la temperatura en las áreas de docencia, administrativas, de gestión, vinculación y otras. Es de vital importancia que los trabajadores de las organizaciones tengan confort térmico, logrando así un equilibrio entre la temperatura del ambiente y la temperatura corporal. Un factor de riesgo lo constituye la percepción de cómo es el espacio físico de las actividades administrativas, de gestión, de vinculación, investigación y otras. Los cuatro factores de prevención encontrados fueron la percepción que se tiene de los espacios físicos destinados a la docencia, los insumos 
disponibles para las actividades de docencia, y la repercusión negativa de la carga académica en las actividades de docencia, administrativas, de gestión, investigación y otras.

\section{RECOMENDACIONES}

Una vez realizado el diagnóstico del caso de estudio y su análisis, se emiten las siguientes recomendaciones:

. Aprovechar el hecho de que el $100 \%$ de los docentes es a tiempo completo para incluirlos de manera activa en el análisis, elaboración, aplicación y seguimiento de los planes de mejora.

- Hacer planes de desarrollo profesional para los docentes, esto generará sentido de pertinencia y que los docentes se planteen objetivos a largo plazo dentro de la organización.

. Se recomienda fomentar espacios donde rescaten valores de amistad, solidaridad y respeto que inciten al compañerismo, entendido éste como el vínculo que se establece entre los integrantes de una organización.

- La motivación es considerada como el motor interno movido por factores externos o internos, que incitan acciones y generan conductas. Se sugiere que las autoridades motiven a sus docentes mediante el reconocimiento, la inclusión en planes y proyectos, que se confíe en su labor y que se genere sentido de pertenencia.

. Es imperativo mejorar las condiciones de temperatura de los lugares de trabajo de la institución que es objeto de estudio, para así incrementar el rendimiento de sus miembros. Se recomienda mejorar la temperatura del aire, colocando aparatos de enfriamiento con gestiones propias, logrando así incentivar el trabajo en equipo y el sentido de pertenencia con la institución.

. Se sugiere ahondar en factores que no han sido tomados en cuenta en esta investigación que inciden en las dimensiones de clima organizacional y desempeño laboral, como por ejemplo el trabajo en equipo, funciones, responsabilidades, actividades de integración, entre otros.

- Se aconseja continuar esta investigación y extenderla hacia las demás carreras de la Facultad con el objeto de buscar coincidencias y realizar planes de mejora a nivel de facultad, facilitando así su implementación y seguimiento. 


\section{REFERENCIAS}

Abreu, J. (2012). Hipótesis, Método \& Diseño de Investigación. International Journal of Good Science, 7(2), 187-197.

Araujo, M. y Guerra, M. (2007). Inteligencia emocional y desempeño laboral en las instituciones de Educación Superior Públicas. Centro de Investigación de Ciencias Administrativas y Gerenciales, 4(2), 132-147.

Camacho, D. (2013). Estrés Térmico en Trabajadores Expuestos al Área de Fundición en una Empresa Metalmecánica, Mariara. Ciencia \& Trabajo, 15(46), 31-34.

Corral, Y. (2009). Validez y confiabilidad de los instrumentos de investigación para la recolección de datos. Valencia, Venezuela: Universidad de Carabobo.

García, M. (2009). Clima Organizacional y su Diagnóstico: Una aproximación Conceptual. Cuadernos de Administración, 42, 43-61.

González, M. y Pérez, I. (2007). Gestión del clima organizacional: una acción deseable en la universidad. Laurus, 13(24), 290-304.

Instituto Nacional de Meteorología e Hidrología. (2019). Boletín climatológico mensual diciembre 2019. Quito, Ecuador: INAMHI.

Jiménez, H. y Mosquera, A. (2017). Clima organizacional y su incidencia en el desempeño laboral de los trabajadores, de los departamentos financieros en entidades públicas (Tesis de grado inédita). Universidad del Pacífico, Quito, Ecuador.

López, P. (2004). Población, muestra y muestreo. Punto cero, 9(8), 69-74.

Martínez, L. (2013). Métodos, técnicas e instrumentos de investigación. Lima, Perú: Multimedia para el desarrollo de trabajos de investigación.

Palaci, F. (2005). Psicología de la Organización. Madrid, España: Pearson Prentice Hall.

Pedraza, E.; Amaya, G. y Conde, M. (2010). Desempeño laboral y estabilidad del personal administrativo contratado de la Facultad de Medicina de la Universidad del Zulia. Revista de Ciencias Sociales, 16(3), 493-505.

Pérez, L.; Soler, S. y Díaz, L. (2009). Ambiente laboral en los policlínicos universitarios. Educación Médica Superior, 23(2), 1-18.

Quintero, N.; Africano, N. y Faría, Elsis. (2008). Clima Organizacional y Desempeño Laboral del Personal Empresa Vigilantes Asociados Costa Oriental Del Lago. Negotium, 3(9), 33-51.

Quiñónez, V. (2013). El clima organizacional y su incidencia en el desempeño laboral de los trabajadores de la Pontificia Universidad Católica del Ecuador - sede Esmeraldas (PUCESE). Quito, Ecuador: Universidad Tecnológica Israel.

Salazar, J.; Guerrero, J.; Machado, Y. Cañedo, R. (2009). Clima y cultura organizacional: dos componentes esenciales en la productividad laboral. Acimed, 20(4), 67-75. 
Salinas, J. (2015). Clima organizacional y su incidencia en el desempeño laboral de los funcionarios de atención al cliente de la Corporación Nacional de Telecomunicaciones del cantón La Libertad - Provincia de Santa Elena - Periodo 2015 (Tesis de grado inédita). Universidad Estatal Península de Santa Elena, Santa Elena, Ecuador.

Segredo, A. (2013). Clima organizacional en la gestión del cambio para el desarrollo de la organización. Revista cubana de Salud Pública, 39(2), 385-393.

Sousa, V.; Driessnack, M. y Costa, I. (2007). Revisión de diseños de investigación resaltantes para enfermería. Rev latino-am enfermagem, 15(3), 1-6.

Torres, M.; Paz, K. y Salazar, F. (2014). Métodos de recolección de datos para una investigación. Revista electrónica de Ingeniería Boletín, 3, 12-20.

Universidad Central del Ecuador. (2009). Campo ocupacional y seguimiento a egresados. Quito, Ecuador: Carrera de Ingeniería Química.

Vega, D.; Arévalo, A.; Sandoval, J.; Aguilar, M. y Giraldo, J. (2006). Diversitas, 2(2), 329349.

Vera, N. y Suárez, A. (2018). Del clima organizacional en el desempeño laboral, el servicio al cliente: Corporación de Telecomunicaciones del cantón La Libertad. Universidad y sociedad, 10(1), 180-186. 